\title{
Polycystic ovary syndrome - an overview
}

\section{K. Muhunthan ${ }^{1}$}

\section{${ }^{1}$ Department of Obstetrics and Gynecology/Faculty of Medicine/University of Jaffna}

\begin{abstract}
Polycystic ovary syndrome (PCOS) is a common condition that has a number of reproductive and general health implications in adults. It is also currently considered the commonest endocrine condition to affect women with an estimated prevalence of $10-15 \%$. Anyhow this prevalence rate depends on the population studied and the diagnostic criteria used.
\end{abstract}

There is significant heterogeneity of presentation, such that signs and symptoms manifest across a spectrum and their severity may vary.

PCOS appears to be underdiagnosed and, as a result, patients may not be managed appropriately. Management of PCOS may require the collaboration of a variety of healthcare professionals.

\section{Introduction}

Polycystic ovary syndrome (PCOS) is a common condition that has a number of reproductive and general health implications in adults. It is also currently considered the commonest endocrine condition to affect women with an estimated prevalence of $10-15 \%$. Anyhow this prevalence rate depends on the population studied and the diagnostic criteria used.

Symptoms of PCOS in adult women include menstrual cycle disturbance and features of hyperandrogenism (hirsutism, acne and alopecia), with associated fertility problems, obesity and psychological issues. There is significant heterogeneity of presentation, such that signs and symptoms manifest across a spectrum and their severity may vary.

The cause of PCOS is unknown, but considerable evidence suggests that it is a complex trait arising from heritable influences, nonheritable intra- and extrauterine environmental factors, variations in insulin resistance, alterations in steroidogenesis/ steroid metabolism, and alternative adaptations to energy excess. $(1,2)$

-18 -
Although the clinical and biochemical presentation of PCOS is heterogeneous, hyperandrogenemia is the most consistent biochemical abnormality, and thus is considered the hallmark of the syndrome.(3)

Various signs and symptoms of hyperandrogenism can manifest prepubertally and the onset of menstrual dysfunction in PCOS typically occurs peripubertally. The syndrome has also been associated with the childhood antecedents of reduced fetal growth, followed by excessive postnatal catch up and premature adrenarche/ pubarche(4,5), suggesting a developmental aspect to its etiology. Moreover, being overweight or obese, a common problem in the pediatric and adult populations, amplifies the clinical severity of the syndrome and increases the risk of metabolic dysfunction.(6)

PCOS appears to be underdiagnosed and, as a result, patients may not be managed appropriately. (6) Furthermore, management of PCOS may require the collaboration of a variety of healthcare professionals, ranging from primary care physicians, gynaecologists, reproductive specialists, endocrinologists, diabetologists, dermatologists, paediatricians, dieticians and psychologists.

\section{Definition and diagnosis}

A diagnosis of PCOS should be made if two out of three criteria are met: the presence of (1) clinical or biochemical features of hyperandrogenism, (2) oligo-ovulation or anovulation and/or (3) polycystic ovaries on ultrasound, once appropriate investigations have been performed to exclude other causes of menstrual disturbance and androgen excess.(7) The diagnosis requires the exclusion of specific underlying diseases of the adrenal or pituitary glands (e.g. hyperprolactinemia, acromegaly, congenital adrenal hyperplasia, Cushing's syndrome and androgen-secreting tumours of the ovary or adrenal gland), which could predispose to similar ultrasound and biochemical 
features and also the exclusion of other causes of menstrual cycle irregularity secondary to hypothalamic, pituitary or ovarian dysfunction.

Polycystic ovaries can exist without clinical signs of the syndrome, expression of which may be precipitated by various factors, most predominantly an increase in body weight. Polycystic ovaries are commonly detected by pelvic ultrasound, with estimates of the prevalence in the general population being in the order of $20-33 \%$.(8) The morphology of the polycystic ovary was also defined as an ovary with 12 or more follicles measuring 2-9 $\mathrm{mm}$ in diameter and/or an increased ovarian volume $(>10 \mathrm{~cm}) .(9)$

The diagnostic criteria for PCOS in adolescence are controversial, primarily because the diagnostic pathological features used in adult women, e.g. acne, irregular menses, and PCOM, may be normal pubertal physiological events.(10) Great caution should be taken before diagnosing PCOS in adolescent girls with clinical features of androgen excess such as hirsutism and biochemical hyperandrogenism if oligomenorrhea has not persisted for more than 2 years. These girls can be considered to be at risk for PCOS. To avoid misdiagnosing physiological pubertal changes as PCOS, deferred diagnostic labeling accompanied by frequent longitudinal re-evaluations of these girls considered to be at risk for PCOS is beneficial and prudent during adolescence.

\section{Epidemiology and ethnic variations}

The prevalence of PCOS in the general population vary considerably between populations that have been studied and the diagnostic criteria being employed.

prevalence of PCOS is probably the same worldwide, although there may be factors that affect expression and presentation.

Type 2 diabetes and insulin resistance have a high prevalence among indigenous populations in South Asia, with a rising prevalence among women. (11)PCOS also appears to run in families, with approximately $50 \%$ of first degree female relatives being affected and an increased risk of metabolic problems in male relatives.

\section{Metabolic abnormalities of PCOS}

The presence of insulin resistance, central obesity, and dyslipidaemia appears to place women with PCOS at higher risk of developing diabetes and cardiovascular disease. Women who are obese, and also many slim women with PCOS will have insulin resistance and elevated serum concentrations of insulin. Both obese and non-obese women with PCOS are more insulin resistant than age and weight matched women with normal ovaries. Thus, there appear to be factors in women with PCOS that promote insulin resistance and that are independent of obesity. Women with PCOS who are oligomenorrheic are also more likely to be insulin resistant than are those with regular cycles, irrespective of their body mass index (BMI). South Asian women should have an assessment of glucose tolerance if their BMI is greater than $25 \mathrm{~kg} / \mathrm{m} 2$ because of the greater risk of insulin resistance at a lower BMI.(12) Further Insulin decreases synthesis of sex hormone binding globulin (SHBG) by the liver, thereby increasing the serum free testosterone concentration, and consequent peripheral androgen action.

It has been suggested that, rather than BMI itself it is the distribution of fat that is important, with android obesity being more of a risk factor than gynecoid obesity.(13) Hence the value of measuring waist circumference, which detects abdominal visceral fat rather than subcutaneous fat. Exercise has a significant effect on reducing visceral fat and reducing cardiovascular risk; indeed, a 10\% reduction of body weight may equate with a $30 \%$ reduction in visceral fat.

\section{Health consequences of PCOS}

Obesity and metabolic abnormalities are recognised risk factors for the development of ischemic heart disease in the general population, and these are also recognised features of PCOS.

There was an increased, though non-significant risk of death due to diabetes but no increased longterm coronary heart disease mortality, although there was evidence of increased stroke-related mortality even after adjustment for BMI.(14) The epidemiology of the long-term risks of PCOS is therefore somewhat conflicting. 


\section{Effect on quality of life}

The symptoms typically associated with PCOS have also been shown to lead to a significant reduction in health-related quality of life. Therefore, any management of the woman with PCOS needs to consider and understand the negative impact this condition may have upon these psychosocial parameters.(15) For example, although the management of hirsuitism may be considered a purely cosmetic issue, excessive facial hair has been shown to be one of the major causes of marked psychological stress in women with PCOS. Infertility and weight issues have also been found to affect other social and psychological parameters.

\section{Management of hyperandrogenism}

The bioavailability of testosterone is affected by the serum concentration of SHBG. High levels of insulin lower the production of SHBG and so increase the free fraction of androgen. Elevated serum androgen concentrations stimulate peripheral androgen receptors, resulting in an increase in 5-alpha reductase activity, directly increasing the conversion of testosterone to the more potent metabolite dihydrotestosterone.

A standardised scoring system, such as the modified Ferriman and Gallwey score, may be used to evaluate the degree of hirsutism before and during treatments. Drug therapy may take 6-9 months or longer before any improvement of hirsutism is perceived. Physical treatments including electrolysis, waxing and bleaching may be helpful while waiting for medical treatments to work. Medical regimens should stop further progression of hirsutism and decrease the rate of hair growth.

First-line therapy has traditionally been the preparation Dianette, which contains ethiny loestradiol in combination with cyproterone acetate. Spironolactone is a weak diuretic with antiandrogenic properties that may be used in women in whom the combined oral contraceptive pill is contraindicated. Drosperinone is a derivative of spironolactone and is contained in the combined oral contraceptive pill Yasmin. In reality all combined oral contraceptive preparations should benefit both the control of the menstrual cycle and suppress hyperandrogenism.
The management of alopecia is difficult but may be stabilised by the anti-androgen preparations mentioned above. Iron deficiency may have an impact on hair loss and so ferritin levels should be assessed.

\section{Management of menstrual problems}

Women with unpredictable, irregular and heavy periods are likely to benefit from cyclical hormone therapy. Furthermore, women with PCOS who experience amenorrhea have unopposed estrogen production from the conversion of androgens and the absence of postovulatory secretion of progesterone. Therefore, the induction of artificial withdrawal bleeds to prevent endometrial hyperplasia is prudent management.(16) The risk of developing endometrial cancer has been shown to be adversely influenced by a number of factors including obesity, long-term use of unopposed estrogens, nulliparity and infertility. It is therefore important that women with PCOS shed their endometrium at least every 3 months. An endometrial thickness greater than $10 \mathrm{~mm}$ in an amenorrheic woman warrants an artificially induced bleed. Another option is to consider a progestogen-secreting intrauterine system, such as the Mirena.

\section{Management of anovulatory infertility}

PCOS accounts for approximately $80-90 \%$ of women with anovulatory infertility. The principles of the management of anovulatory infertility are first to optimise health before commencing therapy (e.g. lose weight in those who are overweight) and then to induce regular unifollicular ovulation. A semen analysis should be performed before ovulation induction therapy is commenced. To ensure a cost-effective approach to treatment, an assessment of tubal patency is appropriate in every woman before commencing therapy.

\section{Obesity and lifestyle}

Various factors influence ovarian function and fertility, the most important being obesity. Monitoring treatment is also harder in obese women because their ovaries are more difficult to see on ultrasound scans, thus raising the risk of missing multiple ovulation and multiple pregnancy. It is suggested that treatment should be deferred 
until the BMI is less than $30 \mathrm{~kg} / \mathrm{m} .(2,17)$ Even a moderate weight loss of $5-10 \%$ of body weight can be sufficient to restore fertility and improve metabolic parameters.

There is no evidence that women with PCOS benefit from a specific diet compared with obese women without PCOS.(18) Meal replacement therapy or low-calorie diets may be appropriate, and it is often helpful to refer to a dietitian. An increase in physical activity is essential, preferably as part of a daily routine. Thirty minutes per day of brisk exercise is encouraged to maintain health. Bariatric surgery is used increasingly because of the global epidemic of obesity and certainly has a role in the management of obese women with PCOS.(16)

\section{Ovulation induction therapies}

Strategies to induce ovulation include oral antiestrogens (principally clomifene citrate [CC]), parenteral gonadotrophin therapy and laparoscopic ovarian surgery. Women with PCOS are at risk of ovarian hyperstimulation syndrome (OHSS) and multiple pregnancy which is why ovulation induction therapy should be carefully monitored with serial ultrasound scans.

\section{Clomifene citrate (CC) therapy}

The anti-estrogen CC has traditionally been used as first-line therapy for anovulatory PCOS.(20) If the patient has not menstruated by day 35 and she is not pregnant, a progestogen-induced withdrawal bleed should be initiated. The dose of CC may be increased to $100 \mathrm{mg}$ if there is no response. CC induces ovulation in approximately $70-85 \%$ of patients and approximately $60-70 \%$ should be pregnant by six ovulatory cycles of therapy.(21)

All women who are prescribed CC should be carefully monitored with ultrasonographic assessment of follicular growth because of the risk of multiple pregnancy, which is approximately $10 \%$. Women with anovulatory infertility who are resistant to anti-estrogens may be prescribed parenteral gonadotrophin therapy or treated with laparoscopic ovarian surgery.

Aromatase inhibitors such as letrozole are an option to be used for ovulation induction.

Vol.30, No.1, July 2018

\section{Gonadotrophin therapy}

Gonadotrophin therapy is indicated for women with anovulatory PCOS who have been treated with antiestrogens if they have failed to ovulate or if they have a response to $\mathrm{CC}$ that is likely to reduce their chance of conception. In order to prevent the risks of overstimulation and multiple pregnancy, a lowdose step-up regimen should be used. Ovulation is triggered with a single subcutaneous injection of human chorionic gonadotropin (hCG) 5000 units, when there has been the development of at least one follicle of at least $17 \mathrm{~mm}$ in its largest diameter.

\section{Insulin-sensitising agents}

It is logical to assume that therapy that achieves a fall in serum insulin concentrations should improve the symptoms of PCOS. The biguanide metformin both inhibits the production of hepatic glucose, thereby decreasing insulin secretion, and also enhances insulin sensitivity at the cellular level. Metformin may improve menstrual frequency and ovulation rate, which may result in a marginal improvement in live birth rate when compared with placebo.

When metformin is added to CC in women with CC resistance, clinical pregnancy rate is increased in both obese and non-obese patients.

\section{Surgical ovulation induction}

An alternative to gonadotrophin therapy for CCresistant PCOS is laparoscopic ovarian diathermy (LOD) (often referred to as 'ovarian drilling' or laparoscopic electrocautery), which has replaced the more invasive and damaging technique of ovarian wedge resection. Laparoscopic ovarian surgery is free of multiple pregnancy risk and OHSS and does not require intensive ultrasound monitoring. In addition, laparoscopic ovarian surgery is a useful therapy for anovulatory women with PCOS who fail to respond to $\mathrm{CC}$ and who persistently hypersecrete $\mathrm{LH}$, need a laparoscopic assessment of their pelvis or who live too far away from the clinic to be able to attend for the intensive monitoring required of gonadotrophin therapy.

\section{Complications of ovulation induction}

Multiple pregnancy and OHSS are the most serious complications that should be avoided in 
ovulation induction treatment. Multiple pregnancy, even twins, is undesirable due to increased risk of perinatal mortality and morbidity. Women with PCOS are at an increased risk of developing OHSS. This occurs if many follicles are stimulated, leading to ascites and pleural and sometimes pericardial effusions, with symptoms of abdominal distension, discomfort, nausea, vomiting and difficulty breathing. Although this condition is rare, it is a potentially fatal complication and should be avoidable with appropriate monitoring of treatment.

\section{IVF in women with polycystic ovaries}

IVF is not the first-line treatment for PCOS, but many patients with the syndrome may be referred for IVF, either because there is another reason for their infertility or because they fail to conceive despite ovulating (whether spontaneously or with assistance); i.e. their infertility remains unexplained.

\section{Pregnancy outcomes}

In addition to anovulation there may be other factors that contribute to subfertility in women with PCOS including the effects of obesity, metabolic, inflammatory and endocrine abnormalities on oocyte quality and fetal development. Women who are obese are also more likely to experience miscarriage and pregnancy complications. It has been found that women with PCOS demonstrated a significantly higher risk of developing gestational diabetes, pregnancy-induced hypertension, preeclampsia, adverse neonatal outcomes and preterm birth.(22) Their babies had a significantly higher risk of admission to a neonatal intensive care unit and a higher perinatal mortality rate, unrelated to multiple births. The potential mechanisms for these problems include obesity, altered glucose metabolism and disturbances in uterine blood flow, and so careful monitoring of pregnancy is required.

\section{Conclusions}

PCOS is one of the most common endocrine disorders. It may present, at one end of the spectrum, with the single finding of polycystic ovarian morphology as detected by pelvic ultrasound. At the other end of the spectrum, symptoms such as obesity, hyperandrogenism, menstrual cycle disturbance and infertility may occur either alone or in combination and have a significant impact on quality of life.

Women with PCOS are characterised by the presence of insulin resistance, central obesity and dyslipidaemia, which appears to place them at a higher risk of developing diabetes and metabolic problems. Encouraging weight loss remains the most effective first-line therapeutic intervention in these women, albeit hard to achieve.

\section{References}

1. Sivayoganathan D, Maruthini D, Glanville JM, Balen AH. Full investigation of patients with polycystic ovary syndrome (PCOS) presenting to four different clinical specialties reveals significant differences and undiagnosed morbidity. Hum Fertil 2011;14:261-5.

2. Barth J, Yasmin E, Balen AH. The diagnosis of polycystic ovary syndrome: the criteria are insufficiently robust for clinical research. Clin Endocrinol (Oxf) 2007;67:811-5.

3. Guastella E, Longo RA, Carmina E: Clinical and endocrine characteristics of the main polycystic ovary syndrome phenotypes. Fertil Steril 2010; 94: 2197-2201.

4. Panidis D, Tziomalos $\mathrm{K}$, Misichronis $\mathrm{G}$, et al: insulin resistance and endocrine characteristics of the different phenotypes of polycystic ovary syndrome: a prospective study. Hum Reprod 2012; 27: 541-549.

5. Conway G, Dewailly D, Diamanti-Kandarakis E, Escobar-Morreale HF, Franks S, Gambineri A, Kelestimur F, Macut D, Micic D, Pasquali R, Pfeifer M, Pignatelli D, Pugeat M, Yildiz BO; ESE PCOS Special Interest Group: The polycystic ovary syndrome: a position statement from the European Society of Endocrinology. Eur J Endocrinol 2014; 171:P1-P29.

6. Rosenfield RL: Identifying children at risk of polycystic ovary syndrome. J Clin Endocrinol Metab 2007; 92: 787-796.

7. Rotterdam ESHRE/ASRM-Sponsored PCOS Consensus Workshop Group. Revised 2003 consensus on diagnostic criteria and long-term health risks related to polycystic ovary syndrome. Hum Reprod 2004; 19:41-7.

8. Michelmore KF, Balen AH, Dunger DB, Vessey MP. Polycystic ovaries and associated clinical and biochemical features in young women. Clin Endocrinol (Oxf) 1999;51:779-86.

9. Balen AH, Laven JSE, Tan SL, Dewailly D. Ultrasound assessment of the polycystic ovary: 
international consensus definitions. Hum Reprod Update 2003;9:505-14.

10. Jeffrey CR, Coffler MS. Polycystic ovary syndrome: early detection in the adolescent. Clin Obstet Gynecol 2007;50:178-87.

11. Wijeyeratne $\mathrm{C}$, Udayangani $\mathrm{D}$, Balen $\mathrm{AH}$. Ethnic specific PCOS. Expert Rev Endocrinol Metab 2013;8:71-9.

12. Wijeyaratne $\mathrm{CN}$, Balen $\mathrm{AH}$, Barth J, Belchetz PE. Polycystic ovary syndrome in south Asian women: a case control study. Clin Endocrinol 2002; $57: 343-50$.

13. Lord J, Wilkin T. Polycystic ovary syndrome and fat distribution: the central issue? Hum Fertil 2002;5:67-71.

14. Wild S, Pierpoint T, McKeigue P, Jacobs $H$. Cardiovascular disease in women with polycystic ovary syndrome at long-term follow-up: a retrospective cohort study. Clin Endocrinol 2000;52:595-600. $128^{\text {a }} 2017$ Royal College of Obstetricians and Gynaecologists Polycystic ovary syndrome

15. Jones GL, Hall JM, Balen AH, Ledger W. Healthrelated quality of life measurement in women with polycystic ovary syndrome: a systematic review. Hum Reprod Update 2008;14:15-25.

16. Fauser BCJM, Tarlatzis BC, Rerbar RW, Legro $\mathrm{RS}$, Balen $\mathrm{AH}$, Lobo $\mathrm{R}$, et al. Consensus on women's health aspects of polycystic ovary syndrome (PCOS): the Amsterdam ESHRE/ASRMSponsored 3rd PCOS Consensus Workshop Group. Hum Reprod 2012;27:14-24 and Fertil Steril

17. Balen AH, Anderson R. Impact of obesity on female reproductive health. British Fertility Society, Police and Practice Guidelines. Hum Fertil 2007;10:195206.

18. Moran LJ, Hutchison SK, Norman RJ, Teede HJ. Lifestyle changes in women with polycystic ovary syndrome. Cochrane Database Syst Rev 2011;(2): CD007506. (b)

19. Scholtz S, Le Roux C, BalenAH. The role of bariatric surgery in the management of female fertility. RCOG Scientific Impact Paper number October 2015;17.

20. Beck JI, Boothroyd C, Proctor M, Farquhar C, Hughes E. Oral anti-oestrogens and medical adjuncts for subfertility associated with anovulation. Cochrane Database Syst Rev 2005;(1):CD002249.

21. Kousta E, White DM, Franks S. Modern use of clomifene citrate in induction of ovulation. Hum Reprod Update 1997;3:359-65.

22. Boomsma CM, Eijkemans MJ, Hughes EG, Visser GH, Fauser BC, Macklon NS. A metaanalysis of pregnancy outcomes in women with polycystic ovary syndrome. Hum Reprod Update 2006;12:673-83. 